Supporting Information

\title{
Chiral Helical Polymer/Perovskite Hybrid Nanofibers with Intense Circularly Polarized Luminescence
}

Biao Zhao ${ }^{\ddagger 1,2}$, Xiaobin Gao ${ }^{\ddagger 1,2}$, Kai Pan $^{2}$, Jianping Deng ${ }^{1,2 *}$

${ }^{1}$ State Key Laboratory of Chemical Resource Engineering, Beijing University of Chemical Technology, Beijing 100029, China

${ }^{2}$ College of Materials Science and Engineering, Beijing University of Chemical

Technology, Beijing 100029, China

*Corresponding author: dengjp@mail.buct.edu.cn

\$B. Zhao and X. Gao contributed equally to this work. 

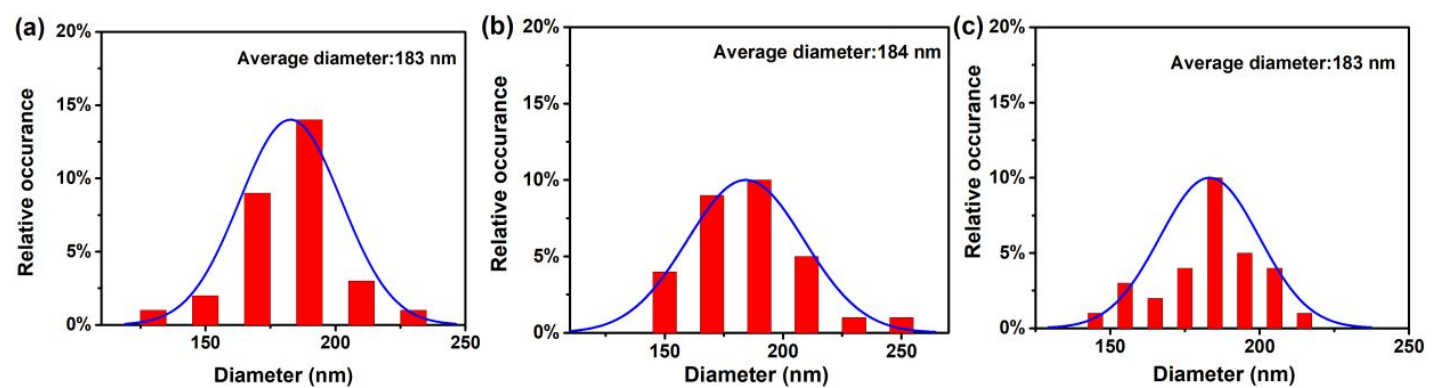

Figure S1. Diameter distributions and average fiber diameter (AFD) of (a) PAN, (b) $S$-PSA/PAN, (c) $S$-PSA/G-PSK/PAN nanofibers.
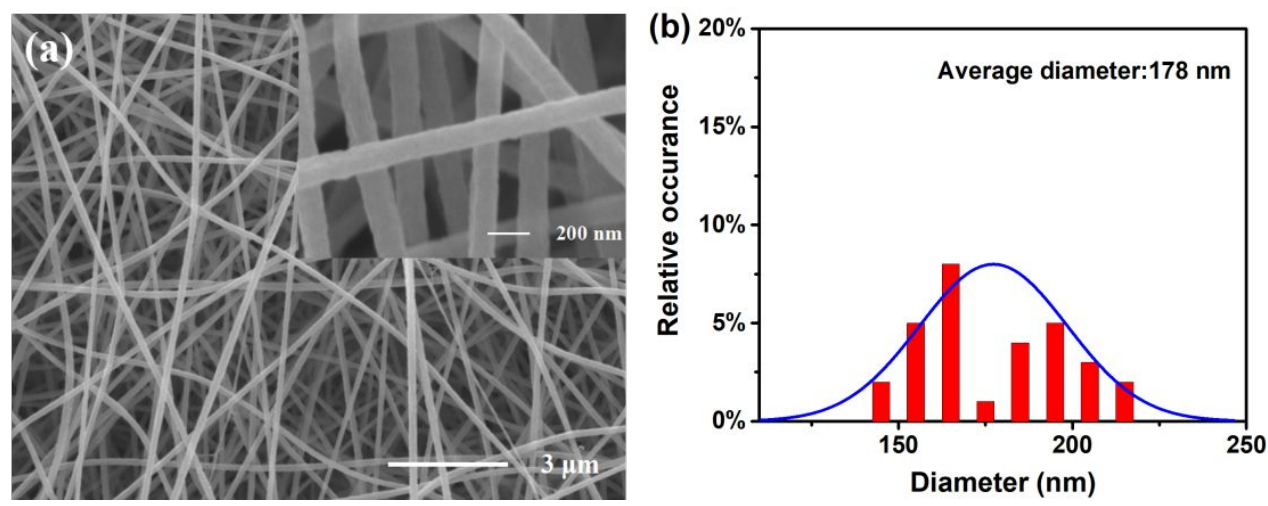

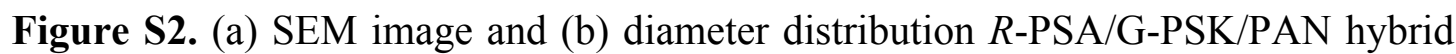
nanofibers.

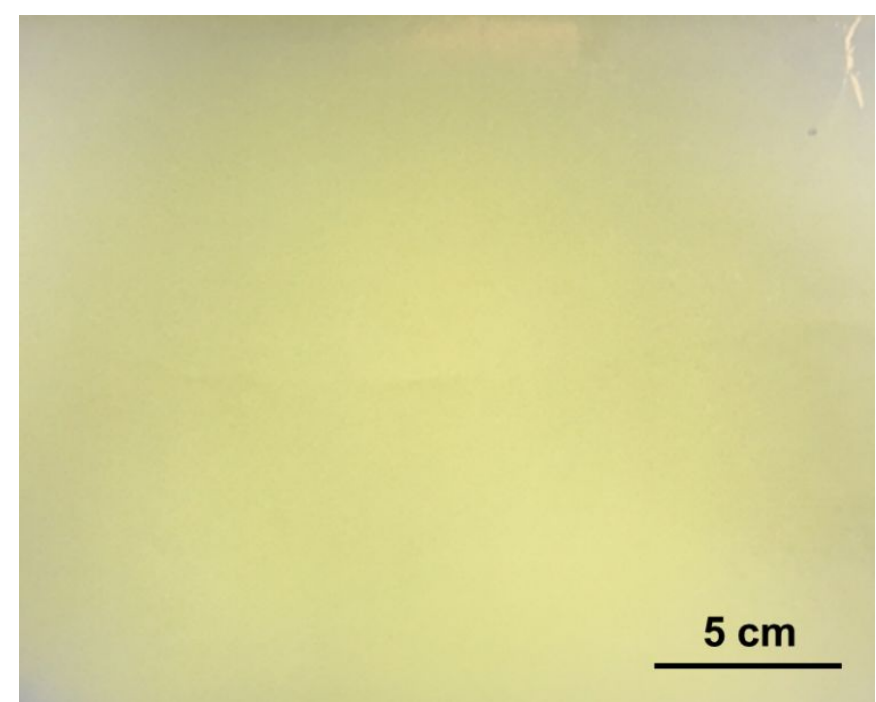

Figure S3. Digital photo of PSA/G-PSK/PAN nanofiber film when the collection distance was $20 \mathrm{~cm}$. 


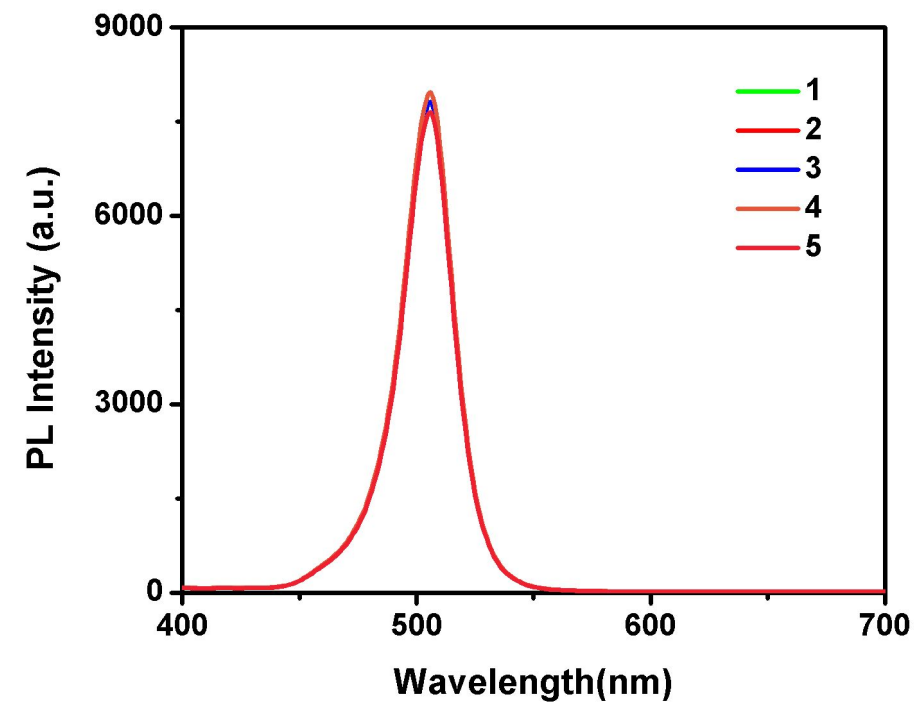

Figure S4. PL spectra of PSA/G-PSK/PAN nanofiber film in different positions.

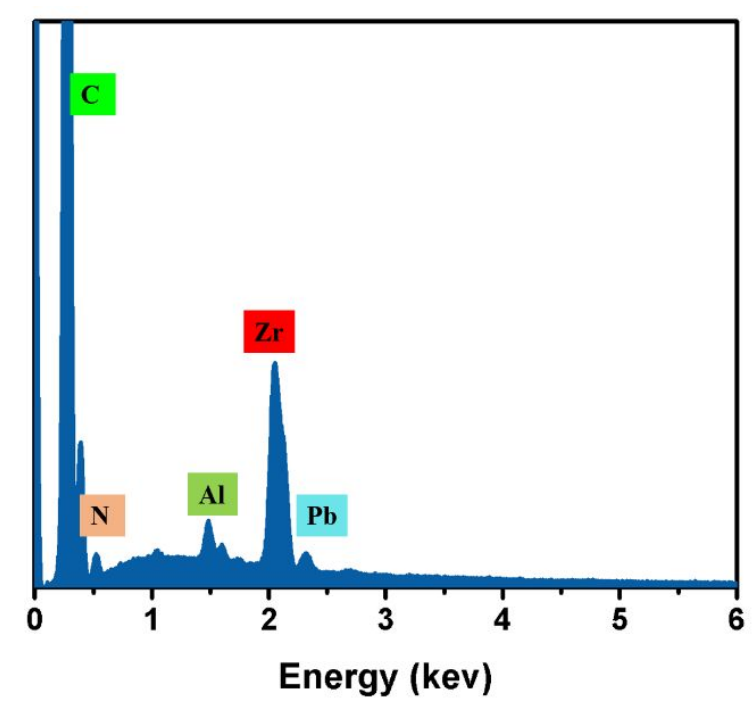

Figure S5. EDS diagram of PAN nanofibers. 
(a)

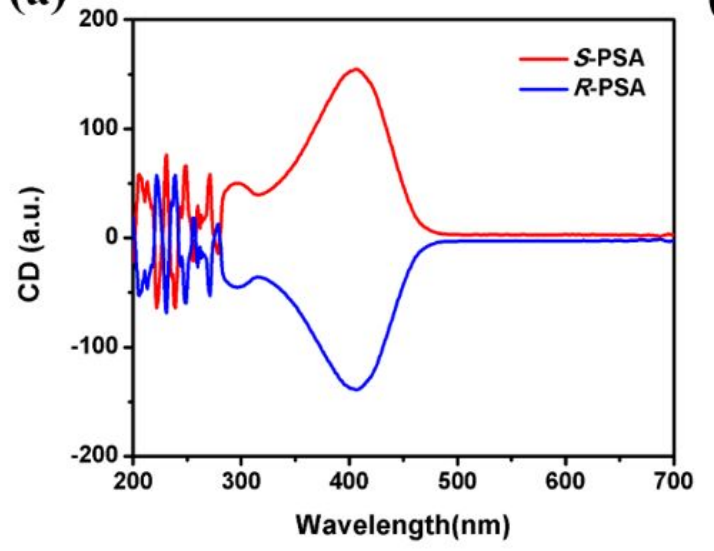

(b)

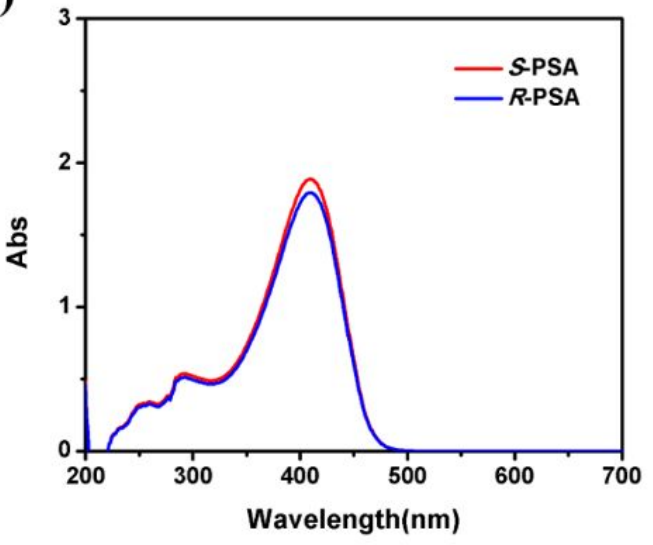

Figure S6. (a) CD and (b) UV-vis spectra of $S(R)$-PSA in toluene solution.

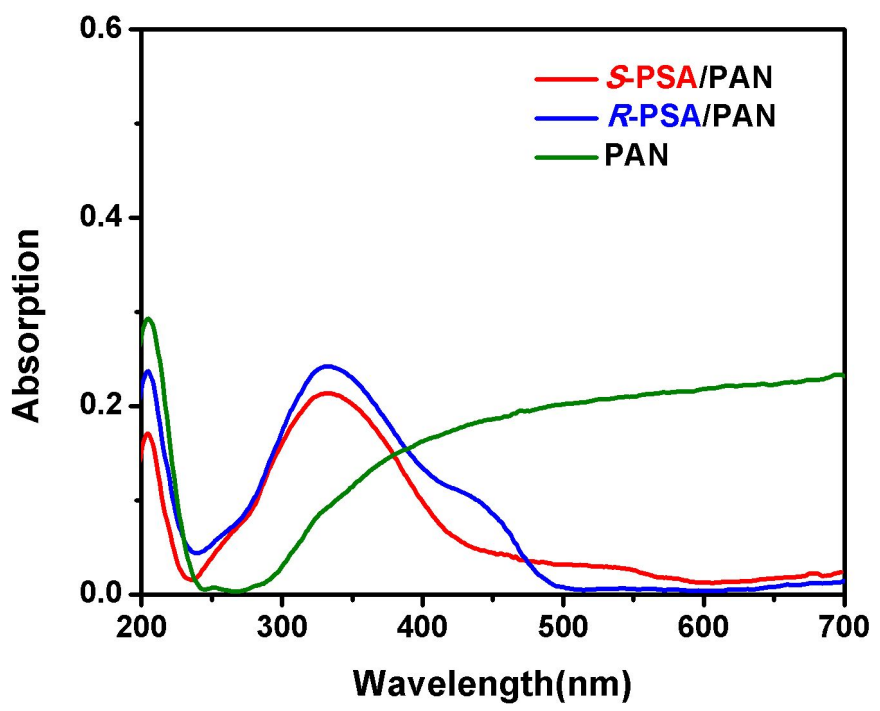

Figure S7. Diffuse-reflectance UV-vis spectra of the electrospinning nanofiber films. 


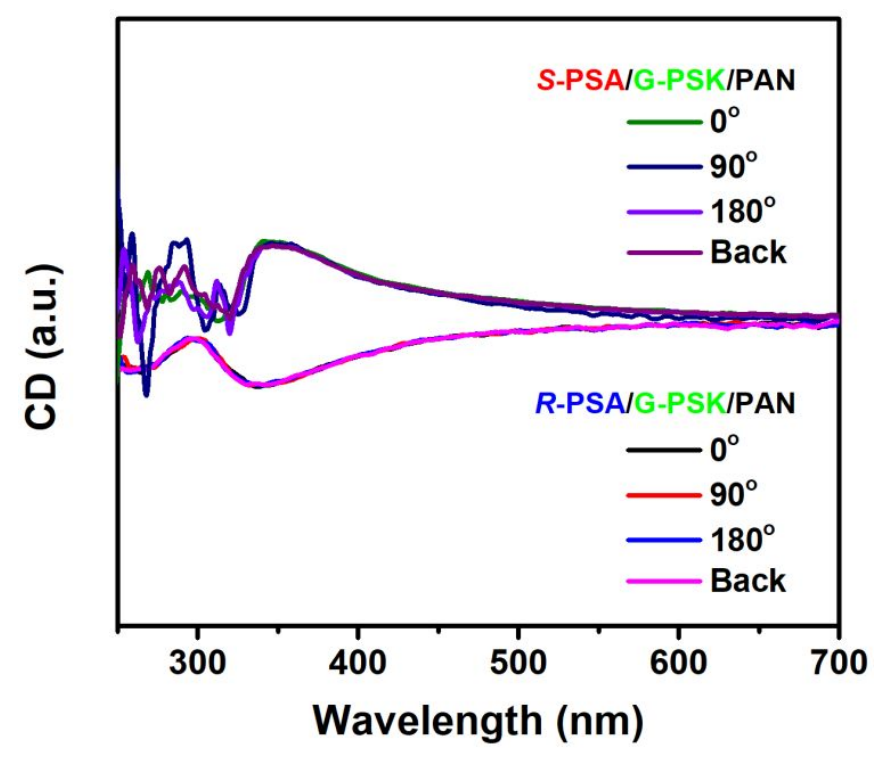

Figure S8. CD spectra of PSA/G-PSK/PAN nanofiber films under different rotating angles.

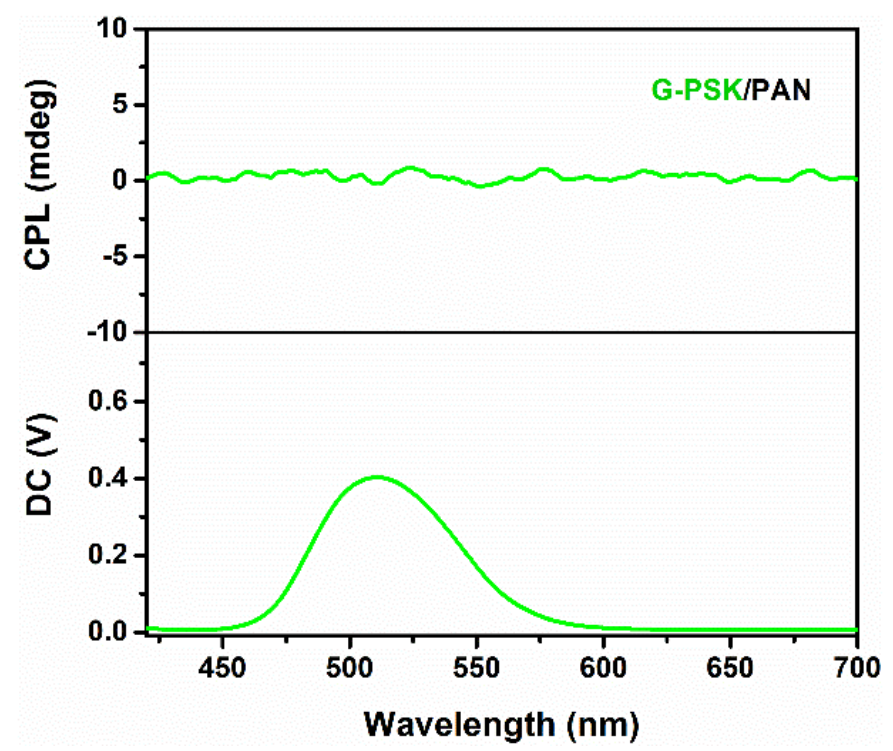

Figure S9. CPL spectra of G-PSK/PAN hybrid nanofibers. 

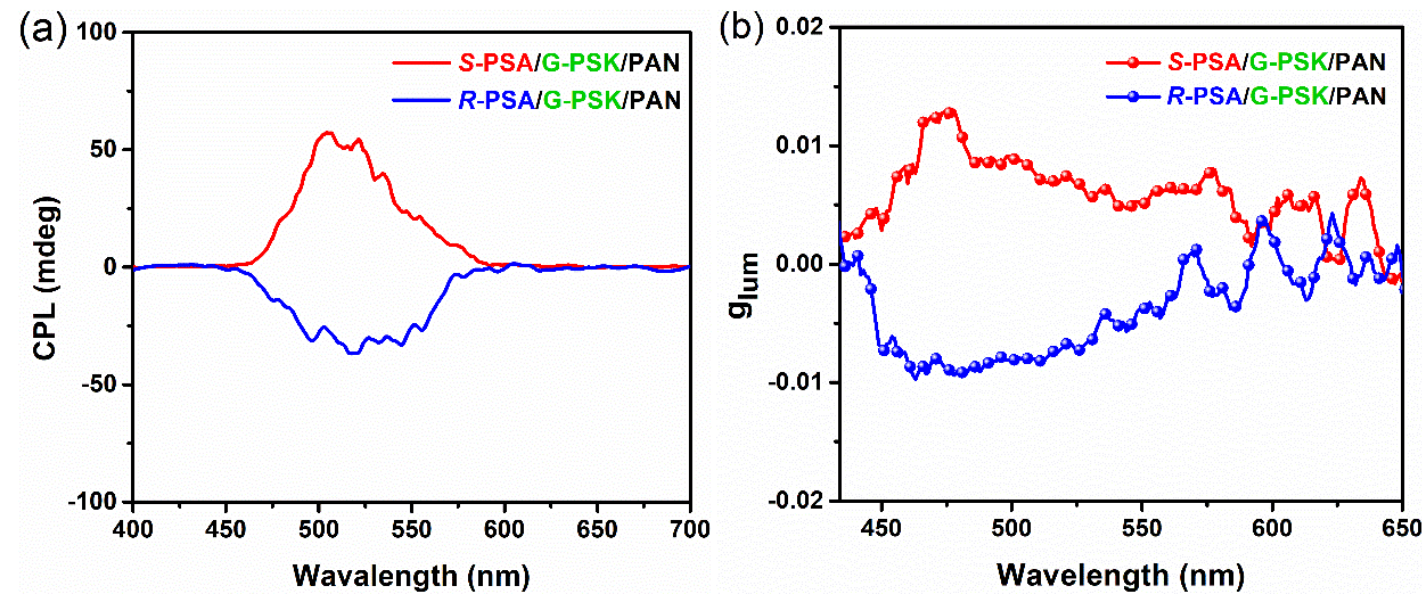

Figure S10. (a) CPL and (b) $g_{\text {lum }}$ spectra of $S(R)$-PSA/G-PSK/PAN hybrid nanofibers with low PSA content $(5 \mathrm{mg} / \mathrm{mL})$.

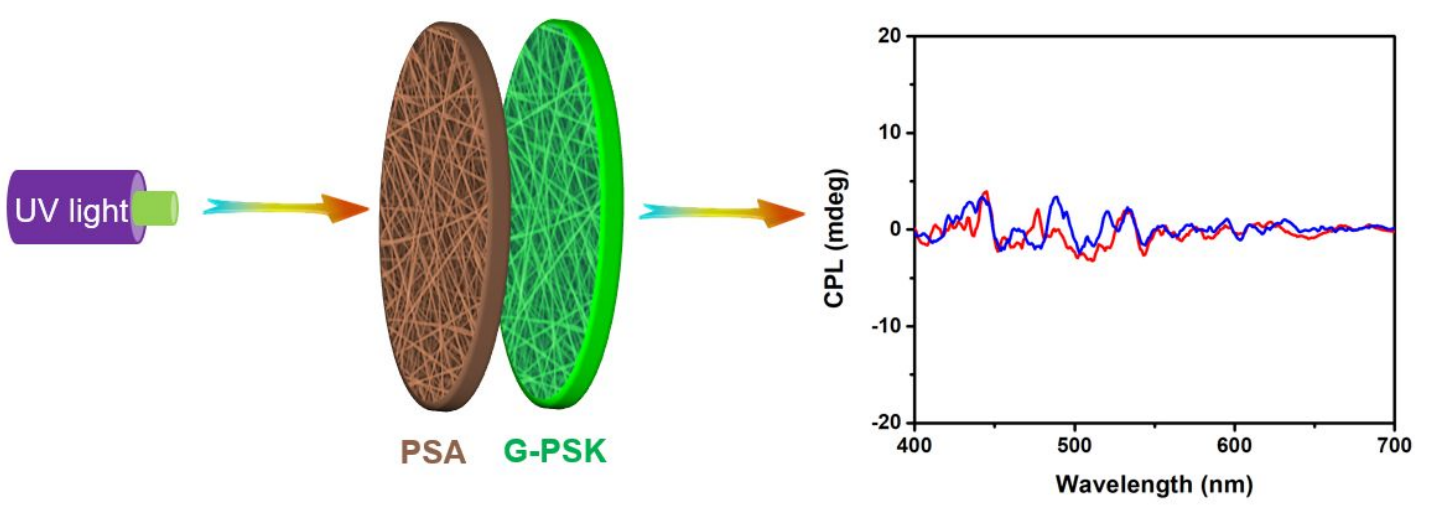

Figure S11. Schematic diagram of CPL measurement of PSA and G-PSK nanofiber films for side-by-side test and the corresponding CPL spectra. 


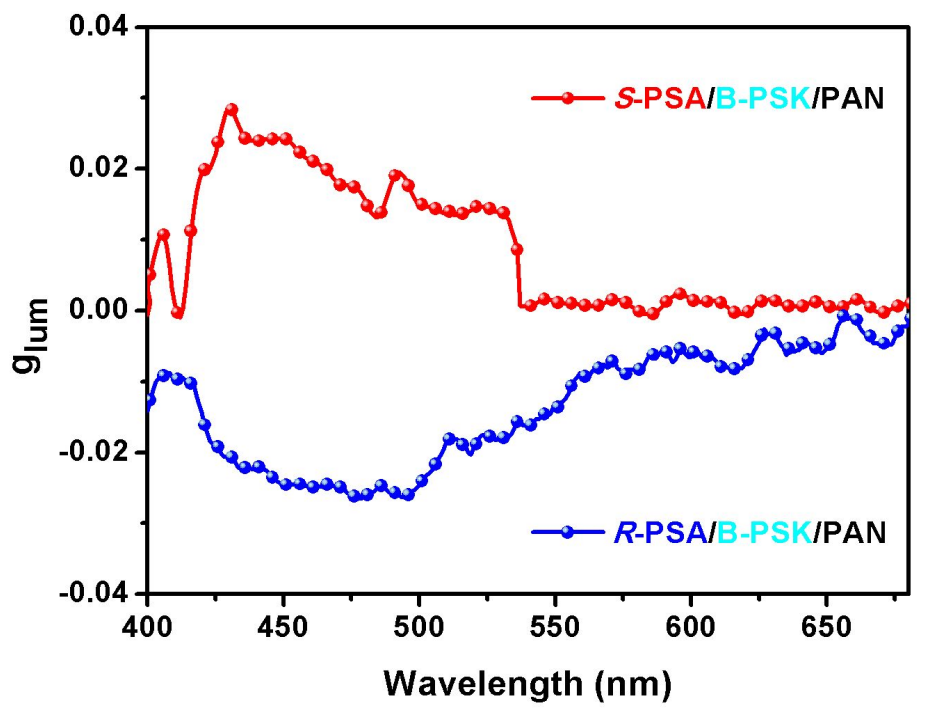

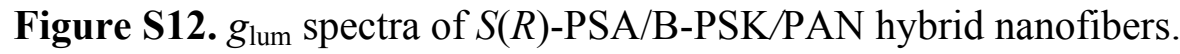
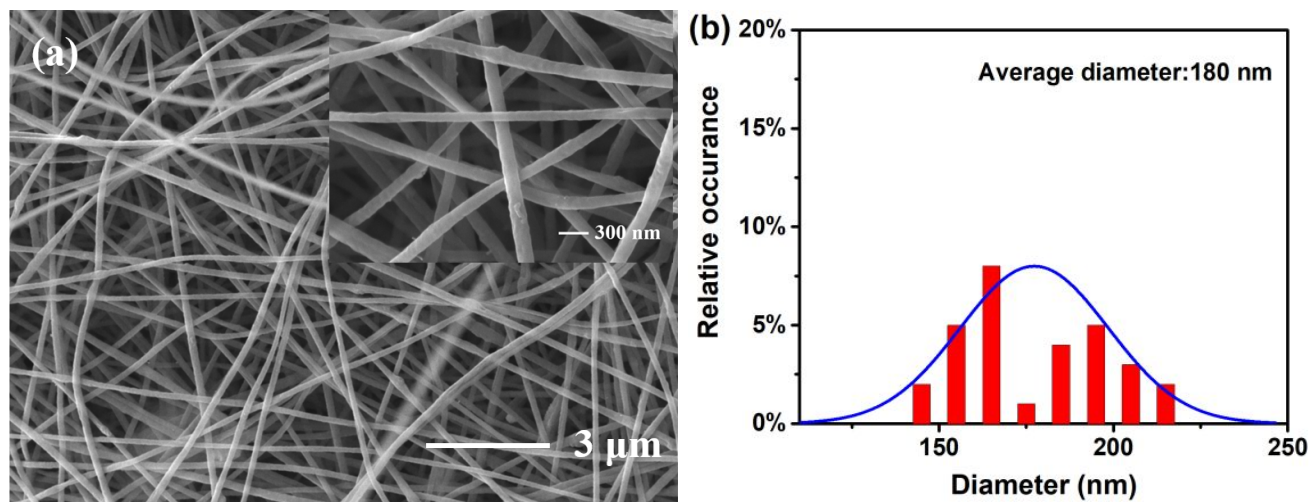

Figure S13. (a) SEM image and (b) diameter distribution of $S$-PM/G-PSK/PAN hybrid nanofibers. 


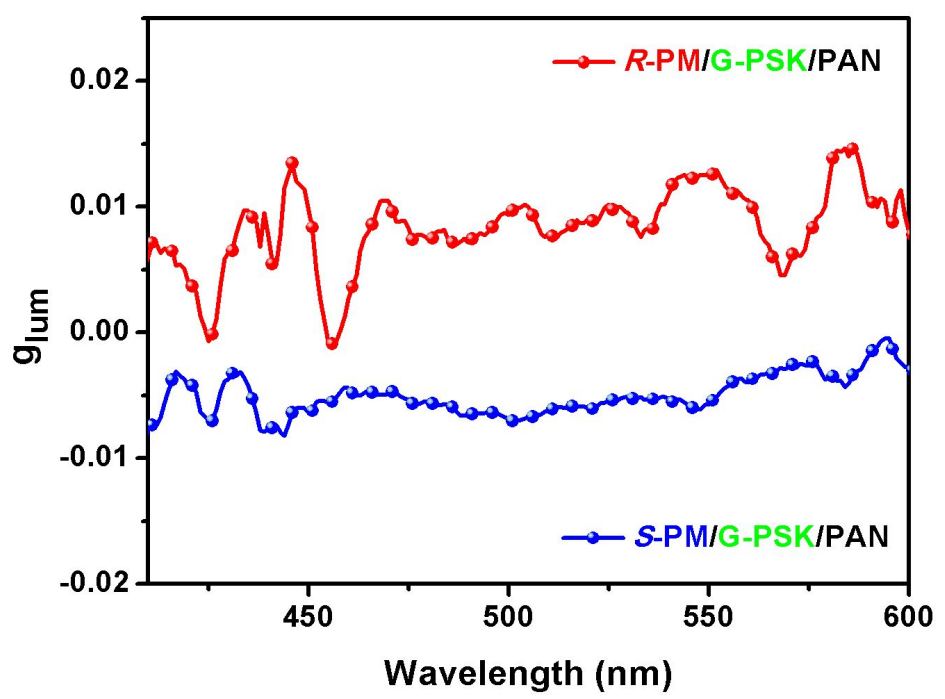

Figure S14. $g_{\text {lum }}$ spectra of $S(R)$-PM/G-PSK/PAN hybrid nanofibers 